\title{
THE QUALITATIVE BEHAVIOR OF A NONLINEAR VOLTERRA EQUATION
}

\section{J. J. $\operatorname{LEVIN}^{1}$}

1. Introduction. The behavior as $t \rightarrow \infty$ of the solutions of the equation,

$$
x(t)+\int_{0}^{t} b(t-\tau) g(x(\tau)) d \tau=f(t) \quad(0 \leqq t<\infty),
$$

has been studied under various assumptions on the given functions $b(t), f(t), g(x)$. A large literature exists for the particular case of (1.1), called the renewal equation, where $b(t) \leqq 0 \leqq f(t)$ and $g(x) \equiv x$; see [1] for references. Motivated by certain problems in heat transfer and superfluidity, the special case of (1.1) where $b(t)=t^{-1 / 2}, f(t)$ is periodic, and $g(x)$ is monotone increasing with $g(0)=0$ has been investigated in [3], [4], [6], [8]. (Except in [3], $f(t) \equiv 1$. In [8], $b(t) \notin L_{1}(0, \infty)$ is taken more generally than $b(t)=t^{-1 / 2}$.) The equation

$$
x^{\prime}(t)=-\int_{0}^{t} a(t-\tau) g(x(\tau)) d \tau \quad\left(\prime=\frac{d}{d t}, 0 \leqq t<\infty\right)
$$

may be converted by integration into the case of (1.1) in which $b(t)=\int_{0}^{t} a(\tau) d \tau$ and $f(t) \equiv x(0)$. It arises in reactor dynamics under hypotheses implying

$$
\begin{aligned}
& a(t) \in C[0, \infty),(-1)^{k} a^{(k)}(t) \geqq 0 \quad(0<t<\infty ; k=0,1,2,3), \\
& g(x) \in C(-\infty, \infty), x g(x)>0 \quad(x \neq 0),
\end{aligned}
$$

and has been studied, e.g., in [2].

If $g(x) \equiv x, f(t) \rightarrow f(\infty) \neq \pm \infty$, and $b(t) \in L_{1}(0, \infty)$ satisfies

$$
\int_{0}^{\infty} \exp [-s t] b(t) d t \neq-1 \quad(\operatorname{Re} s \geqq 0),
$$

then it is known $[7$, p. 58] that any solution $x(t)$ of (1.1) satisfies

$$
\lim _{t \rightarrow \infty} x(t)=\frac{f(\infty)}{1+\int_{0}^{\infty} b(t) d t} .
$$

Received by the editors February 28, 1964.

1 Partially supported by NSF G-24335. 
In this paper a nonlinear version of (1.3) is obtained. The dropping of the linearity assumption $g(x) \equiv x$ causes us to require more of $b(t)$ than (1.2) and more of $f(t)$ than $f(t) \rightarrow f(\infty)$.

Theorems 1 and 2 are concerned, respectively, with the boundedness and asymptotic behavior of the solutions of (1.1).

ThEOREM 1. Let $b(t), f(t)$, and $g(x)$ satisfy

(1.4) $b(t) \in C^{\prime}[0, \infty),(-1)^{k} b^{(k)}(t) \geqq 0 \quad(0 \leqq t<\infty ; k=0,1)$,

(1.5) $f(t) \in C^{\prime}[0, \infty), \int_{0}^{\infty}\left|f^{\prime}(t)\right| d t<\infty$,

(1.6) $g(x) \in C(-\infty, \infty), g(0)=0, g$ monotone nondecreasing.

If $x(t)$ is a solution of (1.1) on $0 \leqq t<\infty$, then

$$
\sup _{0 \leqq i<\infty}|x(t)|<\infty \text {. }
$$

If $f(t)$ also satisfies

$$
\sup _{0 \leqq i<\infty}\left|f^{\prime}(t)\right|<\infty,
$$

then

$$
\sup _{0 \leqq t<\infty}\left|x^{\prime}(t)\right|<\infty .
$$

It may be noted that the hypothesis and the a priori bound obtained in the proof of Theorem 1 guarantee the existence, but not the uniqueness, of a solution of (1.1) on $0 \leqq t<\infty$; see [5].

TheOREM 2. Let $b(t), f(t)$, and $g(x)$ satisfy the hypothesis of Theorem 1 as well as $b(t) \in L_{1}(0, \infty)$ and

(1.10) $b(t)$ is not constant on any interval except, possibly, $b(t) \equiv 0$ on $T \leqq t<\infty$ for some $T$,

$$
\lim _{t \rightarrow \infty} f^{\prime}(t)=0,
$$

(1.12) $g(x)$ is monotone strictly increasing.

If $x(t)$ is a solution of (1.1) on $0 \leqq t<\infty$, then $\lim _{t \rightarrow \infty} x(t)=x(\infty)$ exists and satisfies

$$
x(\infty)+g(x(\infty)) \int_{0}^{\infty} b(t) d t=f(\infty) .
$$

\section{Furthermore,}




$$
\lim _{t \rightarrow \infty} x^{\prime}(t)=0 .
$$

2. Proof of Theorem $1 .^{2}$ Clearly $x^{\prime}(t) \in C[0, \infty)$ and

(2.1) $x^{\prime}(t)=-b(0) g(x(t))-\int_{0}^{t} b^{\prime}(t-\tau) g(x(\tau)) d \tau+f^{\prime}(t)(0 \leqq t<\infty)$.

Define the open, possibly empty, set $g$ by

$$
\mathfrak{g}=\left\{t \mid x(t)>0, x^{\prime}(t)>0, x(\tau)<x(t) \text { for } 0 \leqq \tau<t\right\} .
$$

It is easily seen that

$$
\sup _{0 \leqq i<\infty} x(t) \leqq \max \left(0, f(0), \sup _{t \in \mathcal{I}} x(t)\right),
$$

with equality obtaining if $\sup _{0 \leq t<\infty} x(t) \geqq 0$. For $t \in \mathfrak{g}$, one has, from (1.4), (1.6), (2.1),

$$
\begin{aligned}
x^{\prime}(t) & \leqq-b(0) g(x(t))-g(x(t)) \int_{0}^{t} b^{\prime}(t-\tau) d \tau+f^{\prime}(t) \\
& =-b(t) g(x(t))+f^{\prime}(t) \leqq f^{\prime}(t) \leqq\left|f^{\prime}(t)\right| .
\end{aligned}
$$

Let $g=\cup_{k=1}^{\infty} g_{k}$, where $g_{k}$ are disjoint open intervals and where there may be no terms or only a finite number of terms in the union. If $\left[t_{0}, t_{1}\right] \subset \mathfrak{g}_{k}$ for some $k$, then from (2.3) one has

$$
x\left(t_{1}\right) \leqq x\left(t_{0}\right)+\int_{t_{0}}^{t_{1}}\left|f^{\prime}(t)\right| d t .
$$

It now follows from (1.5) and (2.4) that

$$
\sup _{i \in \mathcal{S}} x(t) \leqq \max (0, f(0))+\int_{0}^{\infty}\left|f^{\prime}(t)\right| d t<\infty,
$$

which, together with (2.2), implies

$$
\sup _{0 \leqq t<\infty} x(t)<\infty .
$$

Define the open, possibly empty, set $D$ by

$$
\mathscr{D}=\left\{t \mid x(t)<0, x^{\prime}(t)<0, x(\tau)>x(t) \text { for } 0 \leqq \tau<t\right\} .
$$

Similar to the preceding paragraph, one successively shows that

\footnotetext{
2 See Added in proof.
} 


$$
\begin{aligned}
\inf _{0 \leqq t<\infty} x(t) & \geqq \min \left(\left(0, f(0), \inf _{t \in \mathbb{D}} x(t)\right),\right. \\
x^{\prime}(t) & \geqq-\left|f^{\prime}(t)\right| \quad(t \in \mathbb{D}), \\
\inf _{t \in D} x(t) & \geqq \min (0, f(0))-\int_{0}^{\infty}\left|f^{\prime}(t)\right| d t>-\infty, \\
\inf _{0 \leqq t<\infty} x(t) & >-\infty,
\end{aligned}
$$

which, together with (2.5), establishes (1.7).

Observing that (1.4) implies $b^{\prime}(t) \in L_{1}(0, \infty)$, one obtains (1.9) as an immediate consequence of (1.6), (1.7), (1.8), and (2.1).

3. Proof of Theorem 2. Define

$$
\hat{x}=\limsup _{t \rightarrow \infty} x(t), \quad \tilde{x}=\liminf _{t \rightarrow \infty} x(t) .
$$

From Theorem 1 it follows that $-\infty<\tilde{x} \leqq \hat{x}<\infty$, and that

$$
\left|x^{\prime}(t)\right| \leqq \beta<\infty \quad(0 \leqq t<\infty)
$$

for some constant $\beta$.

(i) We first prove that

$$
\hat{x}+g(\hat{x}) \int_{0}^{\infty} b(t) d t \leqq f(\infty) .
$$

If $\tilde{x}=\hat{x}$, then obviously $x(\infty)$ exists $(x(\infty)=\hat{x}=\tilde{x})$ and (3.3), wilus the equality sign, is an immediate consequence of (1.1), (1.5), (1.6), and $b(t) \in L_{1}(0, \infty)$. Thus, it remains to establish (3.3) for the case $\tilde{x}<\hat{x}$. Let $\tilde{x}<\bar{x}<\hat{x}$. From the definitions (3.1) it is seen that there exist sequences $\left\{t_{n}\right\},\left\{t_{n}^{*}\right\}$, and $\left\{\epsilon_{n}\right\}$, which depend on $\bar{x}$ such that

$$
\begin{aligned}
& t_{n} \rightarrow \infty, t_{n}-t_{n-1} \rightarrow \infty, x\left(t_{n}\right) \rightarrow \hat{x}, \epsilon_{n} \rightarrow 0 \quad(n \rightarrow \infty), \\
& x^{\prime}\left(t_{n}\right)=0, x\left(t_{n}\right)>\bar{x}, x\left(t_{n}^{*}\right)<\bar{x}, \epsilon_{n+1}>0 \quad(n=1,2, \cdots), \\
& 0<t_{1}^{*}<t_{1}, t_{n-1}<t_{n}^{*}<t_{n} \quad(n=2,3, \cdots), \\
& x(t) \leqq \hat{x}+\epsilon_{n} \quad\left(t_{n-1} \leqq t<\infty ; n=2,3, \cdots\right) .
\end{aligned}
$$

Define

$$
\begin{aligned}
& \gamma_{n}=\frac{1}{\beta}\left(x\left(t_{n}\right)-\bar{x}\right), \\
& \lambda_{n}=\min _{\lambda}\left\{\lambda \mid \lambda>0, x\left(t_{n}-\lambda\right)=\bar{x}\right\} \quad(n=1,2, \cdots) .
\end{aligned}
$$


From (1.6), (3.2), (3.4) it follows that $\lambda_{n}$ exists, $\lambda_{n}>\gamma_{n}>0, t_{n}-\lambda_{n}$ $>t_{n}^{*}$, and

$$
\begin{aligned}
\lim _{n \rightarrow \infty} \gamma_{n} & =\frac{1}{\beta}(\hat{x}-\bar{x})=\bar{\gamma}>0, \\
g(x(\tau)) & \leqq g\left(\bar{x}+\beta\left(\tau-t_{n}+\lambda_{n}\right)\right) \\
& \leqq g\left(x\left(t_{n}\right)\right) \quad\left(t_{n}-\lambda_{n} \leqq \tau \leqq t_{n}-\lambda_{n}+\gamma_{n}\right) .
\end{aligned}
$$

From (2.1) and (3.4) one has

$$
\begin{aligned}
b(0) g\left(x\left(t_{n}\right)\right) & =\left(-\int_{0}^{t_{n-1}}-\int_{t_{n-1}}^{t_{n}-\lambda_{n}}-\int_{t_{n}-\lambda_{n}}^{t_{n}-\lambda_{n}+\gamma_{n}}-\int_{t_{n}-\lambda_{n}+\gamma_{n}}^{t_{n}}\right) \\
& \cdot b^{\prime}\left(t_{n}-\tau\right) g(x(\tau)) d \tau+f^{\prime}\left(t_{n}\right) \\
& =A+B+C+D+f^{\prime}\left(t_{n}\right) .
\end{aligned}
$$

Theorem 1 and (3.4) imply

$$
\begin{aligned}
|g(x(t))| \leqq K<\infty & (0 \leqq t<\infty), \\
\left|g\left(\hat{x}+\epsilon_{n}\right)\right| \leqq K<\infty & (n=2,3, \cdots),
\end{aligned}
$$

for some constant $K$. From (1.4) and (3.9) one notes that

$$
A \leqq-K \int_{0}^{t_{n-1}} b^{\prime}\left(t_{n}-\tau\right) d \tau \leqq K b\left(t_{n}-t_{n-1}\right) .
$$

Similarly, from (1.6) and (3.4), $B$ satisfies

$$
B \leqq-g\left(\hat{x}+\epsilon_{n}\right) \int_{t_{n-1}}^{t_{n}-\lambda_{n}} b^{\prime}\left(t_{n}-\tau\right) d \tau \leqq g\left(\hat{x}+\epsilon_{n}\right) b\left(\lambda_{n}\right)+K b\left(t_{n}-t_{n-1}\right) .
$$

Using the first inequality of (3.7), one finds

$$
\begin{aligned}
C \leqq & -\int_{t_{n}-\lambda_{n}}^{t_{n}-\lambda_{n}+\gamma_{n}} b^{\prime}\left(t_{n}-\tau\right)\left[g\left(\bar{x}+\beta\left(\tau-t_{n}+\lambda_{n}\right)\right)-g\left(x\left(t_{n}\right)\right)\right] d \tau \\
& +g\left(x\left(t_{n}\right)\right)\left[b\left(\lambda_{n}-\gamma_{n}\right)-b\left(\lambda_{n}\right)\right] .
\end{aligned}
$$

As in the inequality for $B, D$ satisfies

$$
D \leqq-g\left(\hat{x}+\epsilon_{n}\right) \int_{t_{n}-\lambda_{n}+\gamma_{n}}^{t_{n}} b^{\prime}\left(t_{n}-\tau\right) d \tau=g\left(\hat{x}+\epsilon_{n}\right)\left[b(0)-b\left(\lambda_{n}-\gamma_{n}\right)\right] .
$$

Equation (3.8) and the preceding inequalities for $A, B, C, D$ imply

$$
\begin{aligned}
0 & \leqq-\int_{t_{n}-\lambda_{n}}^{t_{n}-\lambda_{n}+\gamma_{n}} b^{\prime}\left(t_{n}-\tau\right)\left[g\left(x\left(t_{n}\right)\right)-g\left(\bar{x}+\beta\left(\tau-t_{n}+\lambda_{n}\right)\right)\right] d \tau \\
& \leqq 2 b(0)\left[g\left(\hat{x}+\epsilon_{n}\right)-g\left(x\left(t_{n}\right)\right)\right]+2 K b\left(t_{n}-t_{n-1}\right)+f^{\prime}\left(t_{n}\right),
\end{aligned}
$$


where the second inequality of (3.7), $b\left(\lambda_{n}\right) \leqq b(0)$, and $g\left(\hat{x}+\epsilon_{n}\right)$ $\geqq g\left(x\left(t_{n}\right)\right)$ have also been employed. As the right-hand side of (3.10) tends to zero as $n \rightarrow \infty$, by (1.4), $b(t) \in L_{1}(0, \infty),(1.11)$, and (3.4), it follows that

$$
\lim _{n \rightarrow \infty} \int_{\lambda_{n}-\gamma_{n}}^{\lambda_{n}}\left[-b^{\prime}(s)\right]\left[g\left(x\left(t_{n}\right)\right)-g\left(\bar{x}+\beta\left(\lambda_{n}-s\right)\right)\right] d s=0 .
$$

For simplicity, let us assume momentarily the more stringent form of $(1.10)$ :

$$
b(t) \text { is not constant on any interval. }
$$

We now show that $\lambda_{n} \rightarrow \infty(n \rightarrow \infty)$. Suppose the converse holds. Then there exists a convergent subsequence $\lambda_{n_{k}} \rightarrow \bar{\lambda}<\infty(k \rightarrow \infty)$ of $\left\{\lambda_{n}\right\}$, where $0<\bar{\gamma} \leqq \bar{\lambda}$, by $\lambda_{n}>\gamma_{n}$ and (3.6). From (1.4), (1.6), (1.10'), (1.12), and (3.6) it follows that there exists an interval $I$, independent of $k$, such that: $I$ is of positive length, $I$ is interior to all the intervals $\left[\lambda_{n_{k}}-\gamma_{n_{k}}, \lambda_{n_{k}}\right]$ for $k$ sufficiently large, and on $I$ both $\left[-b^{\prime}(s)\right]$ and $\left[g\left(x\left(t_{n_{k}}\right)\right)-g\left(\bar{x}+\beta\left(\lambda_{n_{k}}-s\right)\right)\right]$ are bounded away from zero. Replacing $\lambda_{n}$ by $\lambda_{n_{k}}$ in (3.11), one sees that the preceding sentence, together with the nonnegativeness of the integrand of (3.11), yields a contradiction to (3.11). Thus $\lambda_{n} \rightarrow \infty$.

By (1.1), (1.4), (1.6), (3.4), and (3.5), one has

$$
\bar{x}+\int_{0}^{t_{n}-\lambda_{n}} b\left(t_{n}-\tau\right) g(x(\tau)) d \tau+g(\bar{x}) \int_{t_{n}-\lambda_{n}}^{t_{n}} b\left(t_{n}-\tau\right) d \tau \leqq f\left(t_{n}\right)
$$

or, equivalently,

$$
\bar{x}+\int_{\lambda_{n}}^{t_{n}} b(s) g\left(x\left(t_{n}-s\right)\right) d s+g(\bar{x}) \int_{0}^{\lambda_{n}} b(s) d s \leqq f\left(t_{n}\right) .
$$

Letting $n \rightarrow \infty$ in (3.13), and noting (1.5), $b(t) \in L_{1}(0, \infty)$, (3.9), and $\lambda_{n} \rightarrow \infty$, one obtains

$$
\bar{x}+g(\bar{x}) \int_{0}^{\infty} b(s) d s \leqq f(\infty) .
$$

As (3.14) has been established for any $\bar{x}$ satisfying $\bar{x}<\bar{x}<\hat{x}$, it follows immediately that (3.3) holds under the hypothesis $\left(1.10^{\prime}\right)$.

The hypothesis (1.10) is composed of two cases: $\left(1.10^{\prime}\right)$ and the case that $b(t)$ is not constant on any interval interior to $0 \leqq t \leqq T$ but $b(t) \equiv 0$ on $T \leqq t<\infty$, for some $T<\infty$. The latter is treated, however, 
much like the former. Instead of $\lambda_{n} \rightarrow \infty$, one obtains, by the above argument,

$$
\liminf _{n \rightarrow \infty}\left(\lambda_{n}-\gamma_{n}\right) \geqq T .
$$

Replacing $\lambda_{n}$ in (3.12) by $\lambda_{n}-\gamma_{n}$, one proceeds as before to obtain (3.3).

(ii) Only slight changes are required in the argument of (i) in order to prove that

$$
\tilde{x}+g(\tilde{x}) \int_{0}^{\infty} b(t) d t \geqq f(\infty) .
$$

For example, in place of (3.4), the sequences $\left\{t_{n}\right\},\left\{t_{n}^{*}\right\},\left\{\epsilon_{n}\right\}$ are now chosen to satisfy the following conditions, where again $\tilde{x}<\bar{x}<\hat{x}$ :

$$
\begin{aligned}
& t_{n} \rightarrow \infty, t_{n}-t_{n-1} \rightarrow \infty, x\left(t_{n}\right) \rightarrow \tilde{x}, \epsilon_{n} \rightarrow 0 \quad(n \rightarrow \infty), \\
& x^{\prime}\left(t_{n}\right)=0, x\left(t_{n}\right)<\bar{x}, x\left(t_{n}^{*}\right)>\bar{x}, \epsilon_{n+1}>0 \quad(n=1,2, \cdots), \\
& 0<t_{1}^{*}<t_{1}, t_{n-1}<t_{n}^{*}<t_{n} \quad(n=2,3, \cdots), \\
& x(t) \geqq \tilde{x}-\epsilon_{n} \quad\left(t_{n-1} \leqq t<\infty ; n=2,3, \cdots\right) .
\end{aligned}
$$

We omit the remaining details.

(iii) It follows immediately from (1.4), (1.6), (3.3), and (3.15) that $x(\infty)$ exists and satisfies (1.13). The result (1.14) is a consequence of $b^{\prime}(t) \in L_{1}(0, \infty),(1.11),(2.1)$, and the existence of $x(\infty)$, which completes the proof.

Note. Equation (1.1) has recently been studied by A. Friedman in On integral equations of Volterra type, Journal D'Analyse Mathématique 11 (1963), 381-413. In this work it is assumed that $f(t)$ is positive and satisfies some monotonicity conditions depending on $b(t)$.

Added in proof. The lemma below is tacitly assumed in the proof of Theorem 1 as given in $\$ 2$. As its proof is not obvious, one (due to S. V. Parter) is supplied here. With the aid of this lemma, inequality (2.2) as well as the step from (2.4) to the inequality which follows it are readily verified. The terms 0 and $f(0)$ appear in these two places because of the condition $x(t)>0$ in the definition of $g$ (this last condition is used in (2.3)).

Lemma. Let $u(t) \in C^{\prime}\left[t_{0}, t_{1}\right],-\infty<t_{0}<t_{1}<\infty$, and let $v(t)$ $=\max _{t_{0} \leqq \tau} u(\tau)$. For $t_{0}<t<t_{1}$, define

$$
\begin{aligned}
& I=\left\{t \mid u^{\prime}(t)>0, u(\tau)<u(t)\left(t_{0} \leqq \tau<t\right)\right\}, \\
& J=\left\{t \mid v^{\prime}(t) \text { exists, } v^{\prime}(t)>0\right\} .
\end{aligned}
$$


Then $I=J, u(t)=v(t)$ and $u^{\prime}(t)=v^{\prime}(t)$ for $t \in I$, and

$$
v\left(t_{1}\right)=u\left(t_{0}\right)+\int_{I} u^{\prime}(\tau) d \tau .
$$

Proof. Let $M=\max \left|u^{\prime}(t)\right|$ on $\left[t_{0}, t_{1}\right]$. From the definition of $v(t)$ it follows readily that $\left|v\left(\tau_{1}\right)-v\left(\tau^{2}\right)\right| \leqq M\left|\tau_{1}-\tau_{2}\right|$ for $\tau_{1}, \tau_{2} \in\left[t_{0}, t_{1}\right]$. Thus $v(t)$ is absolutely continuous on $\left[t_{0}, t_{1}\right]$ and as $v(t)$ is also nondecreasing one has

$$
v\left(t_{1}\right)=u\left(t_{0}\right)+\int_{t_{0}}^{t_{1}} v^{\prime}(\tau) d \tau=u\left(t_{0}\right)+\int_{J} v^{\prime}(\tau) d \tau .
$$

Clearly $I \subset J$ and $u(t)=v(t), u^{\prime}(t)=v^{\prime}(t)$ for $t \in I$.

Conversely, suppose $\bar{t} \in J$. From the definitions of $v(t)$ and $J$ it follows that $u(\bar{t})=v(\bar{t})$. Hence for $t<\bar{t}$ one has $v(\bar{t})-v(t) \leqq u(\bar{t})-u(t)$ which, on dividing by $\bar{t}-t>0$ and letting $t \rightarrow \bar{t}$, implies $u^{\prime}(\bar{t}) \geqq v^{\prime}(\bar{t})$. As it is clearly impossible for $u^{\prime}(\bar{t})>v^{\prime}(\bar{t})$ with $u(\bar{t})=v(\bar{t})$, one has $u^{\prime}(\bar{t})=v^{\prime}(\bar{t})>0$. Also, for $t_{0} \leqq t<\bar{t}$, one has $u(t) \leqq v(t)<v(\bar{t})=u(\bar{t})$. Hence $\bar{t} \in I$ and all of the assertions now follow.

\section{REFERENCES}

1. R. Bellman and K. L. Cooke, Differential-difference equations, Academic Press, New York, 1963.

2. J. J. Levin, The asymptotic behavior of the solution of a Volterra equation, Proc. Amer. Math. Soc. 14 (1963), 534-541.

3. N. Levinson, $A$ nonlinear Volterra equation arising in the theory of superfluidity, J. Math. Anal. Appl. 1 (1960), 1-11.

4. W. R. Mann and F. Wolf, Heat transfer between solids and gases under nonlinear boundary conditions, Quart. Appl. Math. 9 (1951), 163-184.

5. J. A. Nohel, Some problems in nonlinear Volterra integral equations, Bull. Amer. Math. Soc. 68 (1962), 323-330.

6. K. Padmavally, On a nonlinear integral equation, J. Math. Mech. 7 (1958), 533-555.

7. R. E. A. C. Paley and N. Wiener, Fourier transforms in the complex domain, Amer. Math. Soc. Colloq. Publ. Vol. 19, Amer. Math. Soc., Providence, R. I., 1934.

8. J. H. Roberts and W. R. Mann, On a certain nonlinear integral equation of the Volterra type, Pacific J. Math. 1 (1951), 431-445.

UNIVERSITY OF WISCONSIN 